\title{
FRECUENCIA DE LOS HONGOS ASOCIADOS AL DESARROLLO DE MOHOS POSCOSECHA EN EL PEDÚNCULO DE LA PIÑA EN DOS ZONAS DE COSTA RICA
}

\begin{abstract}
Johanny Castro Chinchilla ${ }^{1 / *}$, Gerardina Umaña Rojas*
Palabras clave: Ananas comosus; Penicillium; mohos; frecuencia; poscosecha; cera; cámaras de enfriamiento. Keywords: Ananas comosus; Penicillium; molds; frequency; postharvest; wax; cooling rooms.
\end{abstract}

Recibido: 30/03/17

\section{RESUMEN}

La piña es uno de los principales cultivos de exportación en Costa Rica y la presencia de moho en el pedúnculo afecta su calidad en los mercados internacionales. El objetivo de esta investigación fue determinar en frutos de piña, en la cera aplicada sobre la fruta y en el aire de las cámaras de enfriamiento, la frecuencia de los principales hongos asociados al desarrollo de mohos en el pedúnculo. Se realizó un muestreo mensual de abril de 2012 a marzo de 2013 en empacadoras de Sarapiquí y Puntarenas, Costa Rica. En cada empacadora se muestreó: frutos luego del proceso-comercial que se realiza para exportación y almacenamiento, la cera aplicada sobre la superficie de las frutas y el aire de las cámaras de enfriamiento. Se realizó un análisis de frecuencia de los principales hongos recuperados por muestreo en cada zona, para la cáscara y el pedúnculo, la cera y el aire. Además, se comparó el crecimiento in vitro a $18^{\circ} \mathrm{C}$ de los hongos más frecuentes al finalizar los muestreos. El hongo más frecuente en las cámaras de enfriamiento, el pedúnculo y la cáscara de ambas zonas y en la cera de Sarapiquí fue Penicillium diversum, con valores entre $7 \%$ y $100 \%$. Mientras que en la cera de Puntarenas únicamente destacó

1 Autor para correspondencia. Correo electrónico: johanny.castrochinchilla@ucr.ac.cr
Aceptado: 29/05/17

\begin{abstract}
Frequency of fungi associated to molds in the pedunculus on pineapples from two regions of Costa Rica. Pineapples are one of the top fruits exported from Costa Rica and its quality is affected by molds in the pedunculus at international markets. The objective of this research was to determine in pineapple fruits in the wax applied to the surface of fruits and in the air of cooling rooms, the frequency of the main fungi associated with the development of the molds in the pedunculus. From April 2012 to March 2013 monthly sampling was carried out in 2 packing houses in Sarapiquí and Puntarenas, Costa Rica. In each packing house, fruits were sampled after commercial processing for export and storage and the applied wax on the surface of the fruits and the air of the cooling rooms was sampled too. An analysis of the frequency of the main fungi recovered by the sampling in each zone for the peel and peduncle, the wax and the air was done. Also, it was compared the in vitro growth at $18^{\circ} \mathrm{C}$ of the most frequent fungi at the end of the samplings. The most frequent fungi in cooling rooms, peduncle, peel from both zones and in the wax of Sarapiquí was Penicillium diversum showing the highest frequency, with
\end{abstract}

Universidad de Costa Rica, Centro de Investigaciones Agronómicas, San José, Costa Rica. 
la presencia de un grupo de hongos de micelio blanco. P. purpureogenum, Penicillium sp., Talaromyces calidicanius y Cladosporium sp., fueron microorganismos también recuperados con alta frecuencia tanto en la fruta como en el aire de las cámaras de enfriamiento de ambas empacadoras. $P$. purpureogenum, $P$. diversum y $T$. calidicanius fueron las especies con mayor crecimiento in vitro. Se concluyó que el manejo integrado de $P$. diversum, $P$. purpureogenum y $T$. calidicanius es necesario si se desea evitar el desarrollo de moho en el pedúnculo de la piña.

\section{INTRODUCCIÓN}

La piña (Ananas comosus L.) constituye uno de los principales cultivos de exportación en Costa Rica, con un área sembrada de aproximadamente 43000 has (CANAPEP 2017), que generó en el 2016 la exportación de 1975474 toneladas de fruta fresca y un ingreso de $\$ 881,5$ millones (PROCOMER 1998-2017).

El desarrollo de moho en el pedúnculo de la piña es una de las principales causas de rechazo de la fruta en los diferentes mercados internacionales, situación que disminuye el beneficio económico obtenido por los productores. Se han identificado en Costa Rica diversas especies de hongos asociadas con este problema, entre las que se encuentran Penicillium purpureogenum, Penicillium diversum y un grupo de hongos de micelio blanco (Hgbl), que incluye a las especies Penicillium daleae, Xylaria adscendens y Daldinia eschscholtzii (Castro y Umaña 2015), las cuales fueron recuperadas con alta frecuencia en las diferentes etapas del procesamiento de la fruta.

Tanto las prácticas de manejo del cultivo como la época del año han mostrado tener un efecto significativo sobre los principales microorganismos que causan pérdidas poscosecha, situación que ha sido reportada también en el cultivo values between $7 \%$ and $100 \%$. While in the wax of Puntarenas, only the presence of a group of white mycelium fungi highlighted. P. purpureogenum, Penicillium sp., Talaromyces calidicanius and Cladosporium sp., were microorganisms also recovered with high frequency both in fruit and in the air of the cooling rooms of the both packing houses. $P$. purpureogenum, $P$. diversum and $T$. calidicanius were the species with the higher growth in vitro. It was concluded that the integrated management of $P$. purpureogenum, $P$. diversum and $T$. calidicanius is necessary if development of pineapple molds is to be avoided in the pedunculus.

de mango, al encontrarse variaciones importantes en los microorganismos causantes de pudriciones de frutos del cv. Kent, en función del manejo agronómico del cultivo y de la época del año (Diedhiou et al. 2007). Además, se han encontrado diferencias importantes en la carga microbiológica presentes sobre la superficie de frutos de mango, las cuales dependen de las condiciones climáticas, el estado de desarrollo del fruto y la variedad utilizada (Jha et al. 2010).

En hongos, de las especies Penicillium digitatum, P. italicum y Geotrichum candidum, se identificó un efecto significativo de la temperatura sobre el porcentaje de germinación de esporas y la tasa de crecimiento in vitro, que maximizan ambos parámetros aproximadamente a los $25^{\circ} \mathrm{C}$ (Plaza et al. 2003). Sautour et al. (2002) encontraron diferencias en la tasa de crecimiento in vitro de los hongos Penicillium, Aspergillus y Alternaria, en función de la temperatura y la actividad de agua $\left(\mathrm{a}_{\mathrm{w}}\right)$, lo cual muestra que, además de la frecuencia, también es importante tomar en cuenta las diferencias en la velocidad de crecimiento entre especies de hongos asociados al desarrollo de mohos en poscosecha, la cual podría verse afectada por la 
temperatura de transporte y almacenamiento en el mercado destino.

El objetivo de esta investigación fue determinar en piñas del cultivar "Dorada Extradulce" la frecuencia de los principales hongos asociados al desarrollo de mohos en el pedúnculo, en la cera aplicada sobre la fruta y en el aire de las cámaras de enfriamiento durante un año de producción en 2 zonas de Costa Rica.

\section{MATERIALES Y MÉTODOS}

Se realizó un muestreo mensual entre abril de 2012 y marzo de 2013 en 2 empacadoras de piña localizadas en las provincias de Puntarenas y Heredia, Costa Rica, según la metodología descrita por Castro y Umaña (2015). En cada empacadora se muestreó la cera aplicada sobre la superficie de las frutas y el aire de las cámaras de enfriamiento. Igualmente se analizaron frutos de piña después del proceso requerido para exportación empleado en ambas empacadoras. Esos frutos fueron almacenados durante 18 días a $7^{\circ} \mathrm{C}$ y 3 días a $18^{\circ} \mathrm{C}$ en cámaras de enfriamiento en el Laboratorio de Tecnología Poscosecha del Centro de Investigaciones Agronómicas de la Universidad de Costa Rica. En la cera, en el aire de las cámaras de enfriamiento, en la cáscara y en el pedúnculo de los frutos, Castro y Umaña (2015), determinaron las poblaciones de hongos presentes e identificaron a nivel morfológico y molecular las especies más frecuentes al finalizar los muestreos. Para una revisión más detallada de la metodología empleada para la realización de los muestreos y la identificación de los hongos presentes, se hace referencia al trabajo publicado por los autores mencionados anteriormente.

Se realizó un análisis de frecuencia de los principales hongos recuperados por muestreo en cada sitio, con base en la presencia de cada género y/o especie, en el total de placas Petri utilizadas para cada tejido de la fruta o fase de procesamiento poscosecha (5 para cáscara o pedúnculo, 15 para cera y 15 para cámara de enfriamiento), de acuerdo con la siguiente fórmula:
$F=\frac{\mathrm{Nr}_{*}}{T}{ } 100$, donde $\mathrm{F}=$ frecuencia $(\%) ; \mathrm{Nr}=$ número de placas por muestreo en las que se registró la presencia de cada hongo y $\mathrm{T}=$ total de placas utilizadas para cada tejido de la fruta o fase de procesamiento respectiva.

Adicionalmente, una vez finalizados los muestreos en las empacadoras de cada zona, se seleccionaron los hongos más frecuentes y con cada uno se estableció una prueba de crecimiento in vitro en medio de cultivo papa dextrosa agar acidificado (PDA + Al), que consistió en colocar en el centro de una placa Petri con PDA $+\mathrm{Al}$, aproximadamente $0,5 \mathrm{~cm}^{2}$ de PDA con micelio proveniente de colonias puras de los hongos Penicillium purpureogenum, $P$. diversum, $P$. daleae, Penicillium sp., Talaromyces calidicanius, Cladosporium sp., y Xylaria adscendens. Las placas se almacenaron durante 6 días en una incubadora a $18^{\circ} \mathrm{C}$, con un fotoperíodo de 12 horas. Una vez transcurrido este tiempo, se estimó visualmente en cada placa el porcentaje de cobertura de la superficie del medio de cultivo con el micelio de cada hongo. Se utilizó un total de 10 repeticiones por hongo y cada repetición estuvo constituida por una placa Petri con el medio de cultivo PDA + $\mathrm{Al}$ y el disco con el micelio del hongo respectivo.

Los resultados de las pruebas de crecimiento se presentan como promedios simples con su respectivo intervalo de confianza al $95 \%$, para lo cual, se utilizó el programa estadístico Infostat, versión 2012.

\section{RESULTADOS Y DISCUSIÓN}

El Cuadro 1 muestra la frecuencia de recuperación de los principales hongos aislados en cámaras de enfriamiento y cera de la zona de Puntarenas. El hongo más frecuente en cámaras correspondió a la especie $P$. diversum, aislado en 9 de los 12 muestreos con frecuencias mayores al $7 \%$, el cual alcanzó los valores más altos en mayo y julio de 2012, con 53\% y $72 \%$ respectivamente. El grupo de hongos de micelio blanco (Hgbl) y Cladosporium sp., presentaron una menor frecuencia que $P$. diversum, sin embargo, ambos fueron identificados en más del $50 \%$ de los 
Cuadro 1. Frecuencia mensual de los principales hongos recuperados en cámaras de enfriamiento y muestras de cera de la empacadora de Puntarenas.

\begin{tabular}{|c|c|c|c|c|c|c|c|c|c|c|c|c|c|}
\hline \multirow{3}{*}{ Muestra } & \multirow{3}{*}{ Hongo } & \multicolumn{12}{|c|}{ Muestreo } \\
\hline & & \multicolumn{9}{|c|}{2012} & \multicolumn{3}{|c|}{2013} \\
\hline & & Abr & May & Jun & Jul & $\mathrm{Ag}$ & Set & Oct & Nov & Dic & En & Feb & Mar \\
\hline \multirow[t]{4}{*}{ Cámara } & Penicillium diversum & 0 & 53 & 33 & 72 & 7 & 36 & 7 & 13 & 29 & 0 & 7 & 0 \\
\hline & Penicillium purpureogenum & 0 & 7 & 0 & 6 & 0 & 7 & 0 & 0 & 0 & 0 & 0 & 0 \\
\hline & $\mathrm{Hgbl}$ & 0 & 7 & 11 & 67 & 7 & 7 & 0 & 20 & 21 & 0 & 7 & 7 \\
\hline & Cladosporium sp. & 0 & 0 & 0 & 11 & 0 & 7 & 7 & 7 & 29 & 0 & 7 & 60 \\
\hline Cera & Hgbl & 0 & 27 & 53 & 0 & 13 & 13 & 0 & 0 & 15 & 7 & 0 & 0 \\
\hline
\end{tabular}

**Se resalta en color gris el hongo más frecuente durante el año en cámaras de enfriamiento de Puntarenas.

muestreos, con frecuencias entre $7 \%$ y $67 \%$. La frecuencia de $P$. purpureogenum fue baja, ya que únicamente se recuperó en 3 de los 12 muestreos.

En cera, solamente el grupo de hongos de micelio blanco presentó una alta frecuencia, con mayores porcentajes de recuperación entre mayo y setiembre de 2012, los cuales oscilaron entre $13 \%$ y $53 \%$.
En el Cuadro 2 se muestra que al igual que en la zona de Puntarenas, $P$. diversum presentó una alta frecuencia a lo largo del año en el aire de las cámaras de enfriamiento de Sarapiquí, con valores superiores al $46 \%$ en todos los muestreos. A su vez, $P$. purpureogenum presentó una mayor frecuencia que en Puntarenas, donde se obtuvo aproximadamente un $7 \%$ en los meses en que fue

Cuadro 2. Frecuencia mensual de los principales hongos recuperados en cámaras de enfriamiento y muestras de cera de la empacadora de Sarapiquí.

\begin{tabular}{|c|c|c|c|c|c|c|c|c|c|c|c|c|c|}
\hline \multirow{3}{*}{ Muestra } & \multirow{3}{*}{ Hongo } & \multicolumn{12}{|c|}{ Muestreo } \\
\hline & & \multicolumn{9}{|c|}{2012} & \multicolumn{3}{|c|}{2013} \\
\hline & & Abr & May & Jun & Jul & $\mathrm{Ag}$ & Set & Oct & Nov & Dic & En & Feb & Mar \\
\hline \multirow[t]{5}{*}{ Cámara } & Penicillium diversum & 93 & 46 & 75 & 93 & 93 & 93 & 40 & 71 & 40 & 100 & 60 & 100 \\
\hline & Penicillium purpureogenum & 13 & 42 & 88 & 20 & 7 & 7 & 0 & 0 & 13 & 0 & 0 & 13 \\
\hline & Penicillium sp. & 7 & 67 & 100 & 73 & 40 & 0 & 0 & 50 & 47 & 33 & 13 & 93 \\
\hline & Hgbl & 7 & 25 & 31 & 73 & 27 & 13 & 13 & 0 & 53 & 40 & 33 & 13 \\
\hline & Cladosporium sp. & 7 & 17 & 0 & 20 & 0 & 13 & 7 & 21 & 0 & 7 & 33 & 20 \\
\hline \multirow[t]{4}{*}{ Cera } & Penicillium diversum & 0 & 0 & 7 & 20 & 73 & 40 & 0 & 13 & 47 & 7 & 13 & 7 \\
\hline & P. purpureogenum & 0 & 0 & 0 & 0 & 7 & 0 & 0 & 7 & 20 & 0 & 0 & 0 \\
\hline & Penicillium sp. & 0 & 0 & 47 & 33 & 7 & 0 & 0 & 0 & 7 & 0 & 0 & 27 \\
\hline & Cladosporium sp. & 0 & 0 & 0 & 53 & 0 & 0 & 0 & 0 & 7 & 0 & 20 & 40 \\
\hline
\end{tabular}

\footnotetext{
** Se resalta en color gris el hongo más frecuente durante el año en cámaras de enfriamiento y muestras de cera de Sarapiquí.
} 
recuperado, mientras que en Sarapiquí se obtuvo valores de hasta $88 \%$ en junio de 2012.

Penicillium sp., el cual correspondió a un hongo de micelio verde y base de la colonia amarilla, fue frecuente únicamente en el aire de las cámaras de Sarapiquí (Cuadro 2), en 10 de 12 muestreos realizados, con valores entre 7\% y 100\%. La alta frecuencia del género Penicillium durante el año en cámaras de enfriamiento de ambas empacadoras (Cuadros 1 y 2) y en cera de Sarapiquí, muestra posibles puntos del procesamiento poscosecha de la piña, según las poblaciones de este hongo presentes, las esporas podrían alcanzar la superficie del pedúnculo con el consecuente desarrollo de moho cuando la fruta llega al mercado destino.

La cantidad de hongos aislados con alta frecuencia en la cera, fue mayor en Sarapiquí que en Puntarenas (Cuadros 1 y 2), donde solamente se destacó el grupo de hongos de micelio blanco, posiblemente debido a que en Sarapiquí, según registros de las empacadoras, se procesa mayores volúmenes de fruta que en Puntarenas, esto podría tener un impacto sobre la cantidad y diversidad del inóculo que se acumula en la cera a lo largo del día, la cual es un insumo que, debido a su alto costo económico, se recircula durante el procesamiento de la fruta.

El desarrollo de moho en el pedúnculo de frutos de Puntarenas fue bajo durante el período de evaluación, ya que se obtuvieron los mayores porcentajes en mayo, junio y agosto de 2012, con valores de $3,74 \%, 10 \%$ y $8 \%$ respectivamente, y los principales hongos recuperados en el pedúnculo fueron $P$. diversum y $T$. calidicanius, con frecuencias entre 20\% y 60\% (Cuadro 3). Aunque no se observó desarrollo de moho en la cáscara, ni en la frecuencia de recuperación a partir de este tejido durante el año, la cantidad de hongos presentes fue mayor en comparación con el pedúnculo, ya que adicional a las especies mencionadas, también se presentó una alta frecuencia $P$. purpureogenum del grupo de hongos de micelio blanco, con valores entre $20 \%$ y $40 \%$.

Cuadro 3. Frecuencia mensual de los principales hongos recuperados en el pedúnculo y la cáscara de frutos de piña de la empacadora de Puntarenas.

\begin{tabular}{|c|c|c|c|c|c|c|c|c|c|c|c|c|c|}
\hline \multirow{3}{*}{ Muestra } & \multirow{3}{*}{ Hongo } & \multicolumn{12}{|c|}{ Muestreo } \\
\hline & & \multicolumn{9}{|c|}{2012} & \multicolumn{3}{|c|}{2013} \\
\hline & & Abr & May & Jun & Jul & $\mathrm{Ag}$ & Set & Oct & Nov & Dic & En & $\mathrm{Feb}$ & Mar \\
\hline \multirow[t]{2}{*}{ Pedúnculo } & Penicillium diversum & 0 & 20 & 0 & 0 & $*$ & 0 & 60 & 0 & 0 & 0 & 0 & 0 \\
\hline & Talaromyces calidicanius & 0 & 0 & 20 & 0 & $*$ & 0 & 0 & 0 & 0 & 0 & 20 & 0 \\
\hline \multirow[t]{4}{*}{ Cáscara } & Penicillium diversum & 0 & 20 & 40 & 0 & $*$ & 100 & 20 & 0 & 0 & 40 & 20 & 20 \\
\hline & Penicillium purpureogenum & 0 & 0 & 20 & 20 & $*$ & 20 & 0 & 20 & 0 & 0 & 20 & 0 \\
\hline & Hgbl & 0 & 40 & 20 & 20 & $*$ & 0 & 40 & 20 & 20 & 0 & 0 & 0 \\
\hline & Talaromyces calidicanius & 0 & 0 & 0 & 40 & $*$ & 20 & 20 & 0 & 0 & 40 & 20 & 0 \\
\hline \multicolumn{2}{|c|}{ Porcentaje de moho en el pedúnculo } & 0 & 3,74 & 10 & 1 & 8 & 0 & 0,3 & 0,2 & 0 & 0,5 & 0,17 & 0 \\
\hline
\end{tabular}

*Datos de frecuencia de agosto no disponibles.

**Se resalta en color gris el hongo más frecuente en el pedúnculo y cáscara de frutos de Puntarenas.

$P$. purpureogenum, $P$. diversum y $T$. calidicanius fueron los hongos más frecuentes en el pedúnculo y la cáscara de frutos de Sarapiquí (Cuadro 4), con frecuencias entre $20 \%$ y $100 \%$ durante el año. El porcentaje de moho en el pedúnculo de frutos de Sarapiquí fue mayor que en Puntarenas en la mayoría de los muestreos, al obtenerse un mayor desarrollo de moho en mayo, 
Cuadro 4. Frecuencia mensual de los principales hongos recuperados en el pedúnculo y la cáscara de frutos de piña de la empacadora de Sarapiquí.

\begin{tabular}{|c|c|c|c|c|c|c|c|c|c|c|c|c|c|}
\hline \multirow{3}{*}{ Muestra } & \multirow{3}{*}{ Hongo } & \multicolumn{12}{|c|}{ Muestreo } \\
\hline & & \multicolumn{9}{|c|}{2012} & \multicolumn{3}{|c|}{2013} \\
\hline & & Abr & May & Jun & Jul & $\mathrm{Ag}$ & Set & Oct & Nov & Dic & En & Feb & Mar \\
\hline \multirow[t]{3}{*}{ Pedúnculo } & Penicillium diversum & 60 & * & 80 & 0 & 0 & 0 & 40 & 0 & 0 & 20 & 0 & 80 \\
\hline & Penicilliumpurpureogenum & 100 & $*$ & 20 & 60 & 0 & 0 & 0 & 0 & 0 & 0 & 100 & 80 \\
\hline & Talaromyces calidicanius & 0 & $*$ & 0 & 40 & 0 & 0 & 20 & 0 & 0 & 40 & 40 & 20 \\
\hline \multirow[t]{3}{*}{ Cáscara } & Penicillium diversum & 40 & * & 80 & 0 & 20 & 100 & 0 & 0 & 60 & 20 & 20 & 80 \\
\hline & Penicillium purpureogenum & 40 & $*$ & 0 & 100 & 0 & 0 & 0 & 40 & 80 & 0 & 0 & 0 \\
\hline & Talaromyces calidicanius & 0 & $*$ & 0 & 0 & 60 & 40 & 40 & 60 & 20 & 40 & 40 & 20 \\
\hline \multicolumn{2}{|c|}{ Porcentaje de moho en el pedúnculo } & 4,7 & 24,7 & 5,6 & 12 & 0 & 0 & 0,5 & 0,2 & 0 & 2,3 & 16,9 & 74 \\
\hline
\end{tabular}

*Datos de frecuencia de mayo no disponibles.

**Se resalta en color gris el hongo más frecuente durante el año en el pedúnculo y cáscara de frutos de Sarapiquí.

junio y julio del 2012 y febrero y marzo de 2013, con valores entre $5,6 \%$ y $74 \%$ de cobertura en donde al menos 1 de las 3 especies previamente mencionadas, presentó una frecuencia mayor al 20\%. En la Figura 1 se observan ejemplos de crecimiento en el pedúnculo de los principales

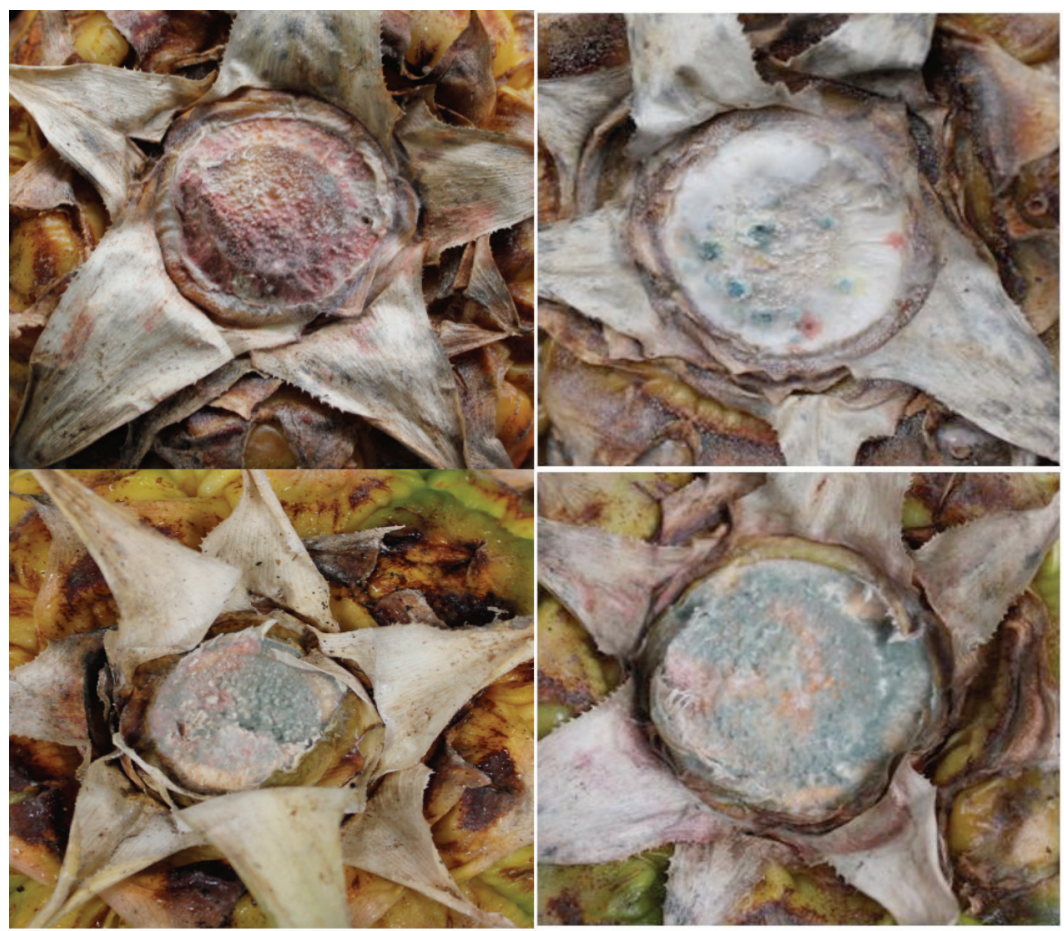

Fig. 1. Crecimiento de los mohos más frecuentes en el pedúnculo de frutos de piña de Puntarenas y Sarapiquí. 
tipos de mohos observados durante los muestreos en ambas empacadoras, donde destaca el color verde, característico de los géneros Penicillium y Talaromyces, y los colores rojizos expresados posiblemente en su mayoría por la presencia de $P$. purpureogenum. Las observaciones realizadas por los autores durante los muestreos permitieron determinar que este tipo de microorganismos crece principalmente de forma superficial sobre el pedúnculo, ya que bajo las condiciones de almacenamiento a las que fueron sometidos los frutos, no se constató la presencia de pudriciones internas en este tejido.

A pesar de que ambas zonas muestreadas se encuentran separadas geográficamente, las especies recuperadas con mayor frecuencia en la cáscara y pedúnculo de la piña fueron similares. Se destacó $P$. diversum, $P$. purpureogenum y $T$. calidicanius como organismos que, independientemente de la época del año, son capaces de permanecer como parte de la carga microbiana de la piña y contribuir significativamente al desarrollo de moho en el pedúnculo, ya que tanto en la cáscara como en el pedúnculo de la piña se encuentran diversas especies de hongos, dentro de las cuales Castro y Umaña (2015) también identificaron a Fusarium proliferatum, Penicillium daleae, Xylaria adscendens, Daldinia eschscholtzii, Microsphaeropsis arundinis, Phoma herbarum y Lasiodiplodia sp. Sin embargo, su frecuencia durante el año en ambas zonas no superó el 10\%, lo cual indica que tienen la capacidad de colonizar el pedúnculo y, eventualmente, contribuir al desarrollo de moho, aunque en menor medida que los géneros Penicillium y Talaromyces.

Cabe destacar que el género Talaromyces, que originalmente fue descrito como el teleomorfo del género Penicillium, actualmente cuenta con 88 especies, entre las que se incluye $T$. calidicanius (Yilmaz et al. 2014), recuperado con alta frecuencia en el pedúnculo y cáscara de ambas zonas. La presencia de esta especie en frutos de piña es un aspecto que podría tener influencia en el manejo poscosecha de los mohos, ya que sexualmente podría generar una mayor variabilidad e influir en la resistencia del hongo a tratamientos como el uso de fungicidas, aspecto que según indican McDonald y Linde (2002), se ve favorecido en aquellos patógenos que combinan ambas estrategias de reproducción. Evidentemente este es un aspecto que debe ser estudiado, para este hongo en particular, en el sistema poscosecha de la piña, además de incluir la respuesta de este hongo a otros tratamientos utilizados convencionalmente en este cultivo para compararla con la de otras especies como $P$. diversum y $P$. purpureogenum.

Las especies $P$. daleae, $X$. adscendens y $D$. eschscholtzii han sido informadas como organismos presentes en ambientes de bosque y suelos, cuya función principal está asociada a la degradación de tejidos (Vidal et al. 1999, Yuyama et al. 2013). Sin embargo, $X$. adscedens también ha sido mencionado como un hongo con capacidad para mantenerse como endofítico en diferentes especies de árboles de bosque tropical lluvioso (Thomas et al. 2016). Este grupo de hongos de micelio blanco, que presentó alta frecuencia principalmente en cámaras de enfriamiento, podría contribuir, aunque en menor medida que el género Penicillium, con el desarrollo de mohos en los frutos de piña, ya que a pesar de no presentar alta frecuencia en el pedúnculo, se observó el desarrollo de este tipo micelio en algunos de los pedúnculos y coronas de los frutos evaluados a lo largo del año, lo cual indica que tienen la capacidad de desarrollarse en estos tejidos y, eventualmente, podrían contribuir significativamente al desarrollo de moho en ausencia de patógenos como Penicillium.

En la Figura 2 se observan diferencias en el crecimiento evaluado como porcentaje de cobertura del medio de cultivo, entre los principales hongos recuperados en el pedúnculo y la cáscara de la piña, donde $P$. purpureogenum, $P$. diversum y $T$. calidicanius fueron los hongos con mayor crecimiento, lo cual muestra la importancia de estos 3 microorganismos causantes de moho en el pedúnculo de la piña, ya que, además de presentar una alta frecuencia durante el año, también crecen con mayor rapidez que otras especies a una temperatura de 


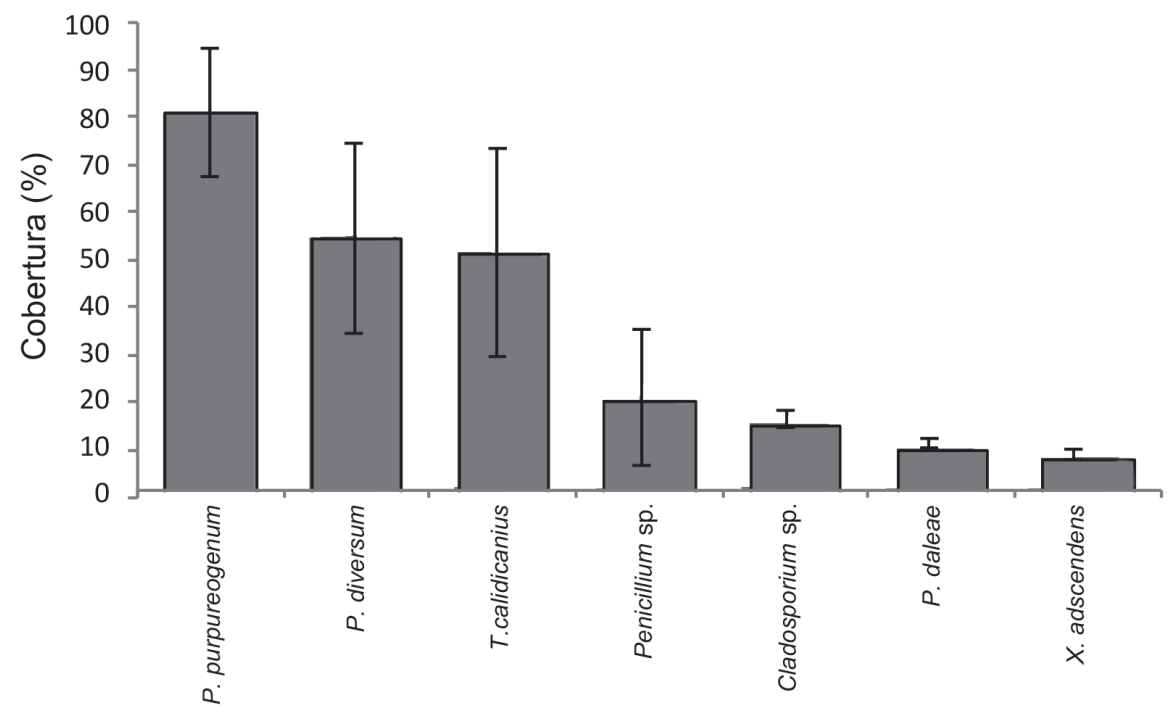

*Barras de error representan el intervalo de confianza al $95 \%$.

Fig. 2. Porcentaje promedio de cobertura del medio de cultivo PDA + Al, con el micelio de los hongos más frecuentes en las empacadoras de Puntarenas y Sarapiquí.

$18^{\circ} \mathrm{C}$, condición a la que usualmente es sometida la fruta en el mercado destino.

$\mathrm{Si}$ bien se encontraron diversos organismos en el pedúnculo y la cáscara de la piña, se destacaron como las especies más importantes $P$. purpureogenum, $P$. diversum y $T$. calidicanius, debido a que presentaron un alta frecuencia durante el año en ambas zonas, con excepción de T. calidicanius, que también fueron recuperados en cámaras de enfriamiento y cera. Además, presentó el mayor crecimiento in vitro, por lo tanto, el manejo integrado de estos microorganismos es importante si se desea disminuir significativamente el desarrollo de moho en el pedúnculo de la piña, sin descartar las demás especies que también presentaron la capacidad de colonizar ese tejido y, aunque en menor medida, contribuyeron en el desarrollo de moho. Como opciones de manejo integrado de estos microorganismos, es importante tomar en cuenta la higiene de las superficies con las que entra en contacto la fruta durante su procesamiento poscosecha, así como las cámaras de enfriamiento, donde el aire que recircula periódicamente es una fuente de esporas de los microorganismos asociados al desarrollo de mohos (Castro y Umaña 2015). Esto también se ha sugerido en cultivos como manzana, donde se considera que la limpieza y desinfección de las cámaras de enfriamiento es fundamental para disminuir el riesgo de infecciones en la fruta causadas por Penicillium expansum (Giraud y Fauré 2000, Morales et al. 2010). Debido a que la cera es una fuente de inóculo de diferentes especies de hongos, principalmente del género Penicillium (Cuadro 2), se deben buscar alternativas de manejo de este insumo, para evitar que esta recircule, o bien, adicionar a la cera sustancias antimicrobianas GRAS (Generally recognised as safe, por sus siglas en inglés), lo cual ha sido citado por Dhall (2013) y Flores-López et al. (2016), como una alternativa para favorecer el control de patógenos en poscosecha. Finalmente, debe tomarse en cuenta que, aunque los fungicidas sintéticos son aún una alternativa para el manejo de enfermedades en poscosecha de piña, su aplicación en los diferentes cultivos es cada vez más cuestionada (Bautista-Baños 2014). Se debe evaluar el efecto de tratamientos alternativos como el uso de calor, radiación ultravioleta (UV-C) y controladores biológicos (Droby et al. 2016, Usall et al. 2016), 
los cuales incluidos dentro de un programa de manejo integrado de mohos, podrían constituirse en alternativas viables para el manejo de patógenos poscosecha en piña.

Con base en los resultados obtenidos en la presente investigación es posible concluir que los hongos $P$. purpureogenum, $P$. diversum y $T$. calidicanius presentan una alta frecuencia durante el año, no solamente en los tejidos de la fruta, sino también en la cera y el aire de las cámaras de enfriamiento, lo cual aunado a su rápido crecimiento in vitro, en comparación con los demás hongos evaluados, evidencia que el manejo integrado de estas especies es importante para lograr un control efectivo del desarrollo de mohos en el pedúnculo de la piña.

\section{LITERATURA CITADA}

Bautista-Baños, S. 2014. Postharvest decay. San Diego, USA. Academic Press. 371 p.

CANAPEP (Cámara Nacional de Productores y Exportadores de Piña). 2017. Estadísticas (en línea) Consultado 22 mar. 2017. Disponible en https://canapep.com

Castro, J; Umaña, G. 2015. Poblaciones e identificación de los hongos causantes de mohos poscosecha en el pedúnculo de la piña, en dos zonas de Costa Rica. Agronomía Costarricense 39(3):61-77.

Diedhiou, PM; Mbaye, N; Dramé, A; Samb, PI. 2007. Alteration of postharvest diseases of mango Mangifera indica through production practices and climatic factors. African Journal of Biotechnology 6(9):1087-1094.

Dhall, RK. 2013. Advances in edible coatings for fresh fruits and vegetables: A review. Critical reviews in food science and nutrition 53:435-450.

Droby, S; Wisniewski, M; Teixidó, N; Spadaro, D; Jijakli, MH. 2016. The science, development, and commercialization of postharvest biocontrol products. Postharvest Biology and Technology 122:22-29.

Flores-López, ML; Cerqueira, MA; Jasso de Rodríguez, D; Vicente, AA. 2016. Perspectives on utilization of edible coatings and nano-laminate coatings for extension of postharvest storage of fruits and vegetables. Food Engineering Reviews 8:292-305.
Giraud, M; Fauré, J. 2000. Assessment of Penicillium risk on pome fruit storage. Integrated Control of Pome Fruit Diseases 23(12):153-158.

Jha, SN; Jaiswal, P; Narsaiah, K; Bhardwaj, R; Sharma, R; Kumar, R; Basediya, AL. 2010. Post-harvest microflora on major cultivars of Indian mangoes. Scientia Horticulturae 125:617-621.

McDonald BA; Linde, C. 2002. The population genetics of plant pathogens and breeding strategies for durable resistance. Euphytica 124:163-180.

Morales, H; Marín, S; Ramos, A; Sanchis, V. 2010. Influence of post-harvest technologies applied during cold storage of apples in Penicillium expansum growth and patulin accumulation: A review. Food Control 21:953-962.

Plaza, P; Usall, J; Teixidó, N; Viñas, I. 2003. Effect of water activity and temperatura on germination and growth of Penicillium digitatum, P. italicum and Geotrichum candidum. Journal of Applied Microbiology 94:549-554.

PROCOMER (Promotora del Comercio Exterior de Costa Rica). 1998-2017. Portal estadístico de comercio exterior (en línea). Consultado 21 mar. 2017. Disponible en servicios.procomer.go.cr/estadisticas/ inicio.aspx

Sautour, M; Mansur, CS; Divies, C; Bensoussan, M; Dantigny, P. 2002. Comparison of the effects of temperature and water activity on growth rate of food spoilage moulds. Journal of Industrial Microbiology \& Biotechnology 28:311-315.

Thomas, DC; Vandegrift, R; Ludden, A; Carroll, GC; Roy, BA. 2016. Spatial ecology of the fungal genus Xylaria in a tropical cloud forest. Biotropica 48(3):381-393.

Usall, J; Ippolito, A; Sisquella, M; Neri, F. 2016. Physical treatments to control postharvest diseases of fresh fruits and vegetables. Postharvest Biology and Technology 122:30-40.

Vidal, S; Salmon, JM; Williams, P; Pellerin, P. 1999. Penicillium daleae, a soil fungus able to degrade rhamnogalacturonan II, a complex pectic polysaccharide. Enzyme and Microbial Technology 24:283-290.

Yilmaz, N; Visagie, CM; Houbraken, J; Frisvad, JC; Samson, RA. 2014. Polyphasic taxonomy of the genus Talaromyces. Studies in Mycology 78:175-341.

Yuyama, KT; Pereira, J; Maki, CS; Ishikawa, NK. 2013. Daldinia eschscholtzii (Ascomycota, Xylareaceae) isolated from the Brazilian Amazon: taxonomic features and mycelia growth conditions. Acta Amazonica 43(1):1-8.

Todos los derechos reservados. Universidad de Costa Rica. Este artículo se encuentra licenciado con Creative Commons Reconocimiento-NoComercial-SinObraDerivada 3.0 Costa Rica. Para mayor información escribir a rac.cia@ucr.ac.cr 